\title{
Self-organized adaptive legged locomotion in a compliant quadruped robot
}

\author{
Jonas Buchli • Auke Jan Ijspeert
}

Received: 29 August 2007 / Accepted: 9 July 2008 / Published online: 29 July 2008

(C) Springer Science+Business Media, LLC 2008

\begin{abstract}
In this contribution we present experiments of an adaptive locomotion controller on a compliant quadruped robot. The adaptive controller consists of adaptive frequency oscillators in different configurations and produces dynamic gaits such as bounding and jumping. We show two main results: (1) The adaptive controller is able to track the resonant frequency of the robot which is a function of different body parameters (2) controllers based on dynamical systems as we present are able to "recognize" mechanically intrinsic modes of locomotion, adapt to them and enforce them. More specifically the main results are supported by several experiments, showing first that the adaptive controller is constantly tracking body properties and readjusting to them. Second, that important gait parameters are dependent on the geometry and movement of the robot and the controller can account for that. Third, that local control is sufficient and the adaptive controller can adapt to the different mechanical modes. And finally, that key properties of the gaits are not only depending on properties of the body but also the actual mode of movement that the body is operating in. We show that even if we specify the gait pattern on the level of the CPG the chosen gait pattern does not necessarily correspond to the CPG's pattern. Furthermore, we present the analytical treatment of adaptive frequency oscillators in closed feedback
\end{abstract}

J. Buchli (凶)

Computational Learning and Motor Control Lab.,

University of Southern California, Ronald Tutor Hall, RTH-417,

3710 S. McClintock Ave, Los Angeles, CA 90089-2905, USA

e-mail: buchli@usc.edu

J. Buchli · A.J. Ijspeert

Biologically Inspired Robotics Group, School of Computer and

Communication Sciences, Ecole Polytechnique

Fédérale de Lausanne, Lausanne, Switzerland loops, and compare the results to the data from the robot experiments.

Keywords Compliant robot - Adaptive controller . Self-organized movement

\section{Introduction}

The current state of the art in robotic legged locomotion does not compare in any means to legged locomotion observed in the animal kingdom. The fact that there already has been a large research effort devoted to robotic locomotion indicates that it is not just a matter of better implementing well-known basic principles. Rather, it points to a more fundamental problem, namely that the we do not know all the required basic principles. Hence the way robots are built and controlled simply do not support fast, efficient, robust and agile locomotion.

While it has been realized for a while now that (1) compliance might be a key factor, (2) the body-environment system is self-organizing so locomotion can not be understood in isolation, and (3) a high degree of adaptation is required, systems which implement all these key factors are rare. In this article, we show experiments including to some extent all the three principles.

An important concept from neurobiology which has stimulated a lot of research in robotics is the concept of the Central Pattern Generator (CPG). It has been realized that in vertebrates the neural centers generating the high-dimensional coordinated gait patterns are located in a distributed fashion in the spine rather than in higher brain centers (Grillner 1985). The CPG is under modulatory control by the brain. Subsequently, many researchers have taken up the ideas and many studies ranging from theoretical (Schöner et al. 1990; 
Collins and Richmond 1994) to experimental (e.g. Tsuchiya et al. 2002; Kimura et al. 2004; Morimoto et al. 2006; Endo et al. 2005; Ijspeert 2001; Crespi and Ijspeert 2006; Ijspeert et al. 2007) about the use and properties of CPGs have been done. One of the main motivations of preferring CPGs over other trajectory generation methods is their limit cycle behavoir and attractor properties. Yet, most applications of CPGs are on stiff, fully actuated robots where the trajectories given by the CPG are accurately followed by help of high-gain control (see e.g. Kimura and Fukuoka 2004 for an exception). It is very unlikely that CPG work like this in nature. As observed by Marc Raibert, the central nervous system does not control the body, it can only make suggestions (Raibert and Hodgins 1993). In this contribution, we show exactly the application of Raibert's insight. There have been difficulties in putting these ideas to realization. We argue that parts of these are due to an inappropriate methodology which is unable to deal with such compliant and selforganizing systems in a methodological fashion. We propose to use non-linear systems (oscillators) where we can exploit the intrinsic self-organizing capability of such systems. The desired controller dynamics are designed as an attractor state of the system. The attractor dynamics are determined by the body-environment system.

The controllers that we use in this article are loosely inspired by the CPG concept in that they are dynamical systems which can show their own coordinated spatio-temporal patterns. The controller consists of adaptive frequency oscillators. We illustrate their novel property of being truly adaptive, in that the controllers tune key parameters such as frequencies to match the body properties of the robot.

We show the use of the adaptive controllers on the compliant under-actuated quadruped robot PUPPY II which has strong body dynamics. As previously shown (Iida et al. 2005), PUPPY II can show many different modes of locomotion, dependent of many critical parameters. Such modes include bounding (alternating) gaits, jump gaits, gaits with higher periodicity, etc. In the previous studies these parameters where found/tuned manually (Iida et al. 2005) and emphasized the need to adapt to the different environments and body conditions without demonstrating how. In this contribution we show a first attempt at such an adaptive controller, and we take the concept further and even allow some of the parameters to be determined by the robot-environment system itself, namely phase (i.e. gait pattern) and the frequency. This means that we exploit the self-organization capabilities of the body-environment system to simplify the control problem and improve the controller performance.

More precisely, we investigate on the following four main topics: (1) Robustness-One central feature which makes attractor dynamics an interesting tool to design controllers or, in the wider sense, behaviors for a robot is that attractor dynamics is very robust under noisy conditions and perturbations. We show how concepts earlier only presented in simulations (Buchli and Ijspeert 2004b; Buchli et al. 2005) are very robust with the noisy signals of real sensors and therefore the simple systems developed in theory and simulations can readily be implemented in real world systems. (2) Self-organization of gait-We show that even if we specify the gait pattern on the level of the CPG (by full coupling) the chosen gait pattern (measured by the foot-fall pattern) does not necessarily correspond to the CPGs pattern. There are modes which are preferred due to the mechanics and its coupling to the environment. We show that controllers based on dynamical systems as we present are able to "recognize" such mechanically intrinsic modes of locomotion, adapt to them and enforce them. (3) Tracking of resonant frequency - It is well known that a body, of an animal or robot, has specific resonant frequencies (e.g. from elasticity or pendulum dynamics). Less studied however is how these frequencies depend not only on the spring coefficients of the compliant elements, but also on the posture and type of gait. We will show that they do depend on the posture and type of gait and we will present an adaptive controller which can account for this dependence. (4) Theory-Finally, we show that we have a theory to understand adaptive frequency oscillators in feedback loops. Generally speaking, this gives us a tool to work with resonant dynamics of any type of system, and therefore adaptive frequency oscillators can be used to either find them or avoid them.

This paper is organized as follows. We first briefly review related work. We then introduce the adaptive frequency oscillator and show the structure of the adaptive controller (Sect. 2). Then the hardware setup is introduced (Sect. 3), followed by presentation of the results which is split into two parts. Firstly we show experimental results of adaptive controllers on a compliant robot, highlighting different important aspects and findings (Sect. 4), and secondly, we show theoretical results about the convergence behavior of such adaptive controllers (Sect. 5).

Related work Early work on spring dynamics in animal models can be found in Full and Koditscheck (1999) and important contributions from the robotics community showing self-stabilization and simple control in robot with appropriate body dynamics can be found in McGeer (1990), Collins et al. (2005), Kimura and Fukuoka (2004), Cham et al. (2004), Raibert (1990), Iida et al. (2005).

Recent studies on mammals have shown that many muscle groups in the mammalian legs effectively are anti-gravity muscles and do not directly contribute to locomotion (Fischer and Blickhan 2006). These studies have sparked quite some theoretical treatment of locomotion with legs with spring-like properties (Geyer et al. 2004).

While the idea to "adapt" to resonant body dynamics is not new, usually the systems employed work more in a reactive fashion than truly adaptive, i.e. their parameters remain 
constant, and especially do not reflect the body parameters after the adaptation process. See Buchli et al. (2006c) for a more in-depth discussion of this issue. In the frame of oscillators this typically means that synchronization (i.e. phase locking) is exploited. However, as we will explain later, the adaptive frequency property that we exploit in our study is a fundamental extension of synchronization or phase locking. An interesting example is Verdaasdonk et al. (2007). While still not truly adaptive, the remarkable result of this study is that the locking region is extremely large. In Iwasaki and Zheng (2006), Futakata and Iwasaki (2008) the authors analyze oscillators in feedback with linear plants. Although the employed oscillators are also not adaptive oscillators, it is to our knowledge the only contributions which systematically employ linear systems theory to the analysis of oscillators in feedback with plants. In Reddy et al. (2000) the authors analyze Hopf oscillators, with feedback. Again the oscillators are traditional non-frequency adaptive oscillators. The feedback is either linear, pure time delay and/or non-linear. Under the limits of a comparison of a pure time shift system to a minimum phase linear system the results do align with our analysis.

The use of adaptive frequency oscillator for adapting to resonant body dynamics has been first presented in Buchli and Ijspeert (2004b). Further result on a more complex robot simulation has been presented in Buchli et al. (2005). In Buchli et al. (2006b) we presented first preliminary results of the adaptation on a real robot. Here we present more detailed data for different experiments. The theoretical understanding of adaptive frequency oscillators has been advanced in Righetti et al. (2006) by proving its convergence and first results on the treatment of AFOs in feedback loops are shown in Buchli et al. (2006b).

\section{Adaptive controller}

In this section we introduce the dynamical systems and methods that we use to construct the controllers of the robot. The controller consists of one or several oscillators mutually coupled and influenced by sensory information.

The building blocks of our controllers are oscillators. More specifically, we use the Hopf oscillator (Hopf 1942):

$\dot{\mathbf{q}}=\mathbf{F}_{H}\left(\mathbf{q}_{i}\right)+\mathbf{p}=\left[\begin{array}{c}\left(\mu-\left(q_{1}^{2}+q_{2}^{2}\right)\right) q_{1}+\omega q_{2} \\ \left(\mu-\left(q_{1}^{2}+q_{2}^{2}\right)\right) q_{2}-\omega q_{1}\end{array}\right]+\mathbf{p}$

where, $\mathbf{q}=\left[q_{1}, q_{2}\right]$ are the state variables, $\sqrt{\mu}$ is the steady state amplitude, and $\omega$ the intrinsic frequency of the oscillator. $\mathbf{p}=\left[p_{1}, p_{2}\right]$ is an additive input to the oscillator. The nice feature of this oscillator is its harmonic limit cycle, i.e. we can write the steady state solution of the system (1) without perturbation $(p=0)$ by $q_{1}(t)=\sqrt{\mu} \cos \left(\omega t+\phi_{0}\right)$, $q_{2}(t)=\sqrt{\mu} \sin \left(\omega t+\phi_{0}\right)$, where $\phi_{0}$ is the angle of the initial condition $\mathbf{q}(0)$. The harmonic limit cycle allows to analytically determine the phase sensitivity and this allows to determine the phase relationship to other oscillators for example (Buchli and Ijspeert 2004a).

The oscillators receive input from the sensors of the robot. The form of the input is different for different experiments. In general the sensor values are converted into a zero-mean signal by a high-pass filter and multiplied by a coupling constant to achieve a suitable input range (typically values are between -1 and 1$)$. In other words $p_{1}=$ $K_{s} s(t)+\cdots$ where $s$ is the (filtered) sensor value.

Adaptive frequency oscillators In some experiments the oscillators will be extended to adaptive frequency oscillators. Adaptive frequency oscillators have been introduced in Buchli and Ijspeert (2004b) and generalized and treated in more detail in Righetti et al. (2006).

The idea behind adaptive frequency oscillators is that we endow the oscillators with the capability to tune their intrinsic frequency to the frequency of a perturbation. This can be achieved by posing the following general rule as differential equation for the intrinsic frequency (or a parameter which tunes the intrinsic frequency):

$\dot{\omega}=-\tau p_{1} \frac{q_{2}}{\sqrt{q_{1}^{2}+q_{2}^{2}}}$

where $\tau$ is an adaptation constant. It is important to realize, that the adaptive frequency property that we exploit in our study is a fundamental extension of synchronization or phase locking. (1) The adaptation process changes the intrinsic frequency and not only the resulting frequency, (2) the adaptation generally has an infinite basin of attraction (i.e. for every initial condition $\omega(0)$ it will converge to a frequency $n \omega_{F}$ as opposed to the limited range in which synchronization can take place, also known as Arnol'd tongues structure, Arnol'd 1983; Pikovsky et al. 2001), (3) the frequency stays encoded in the system when the input is removed (e.g. set to zero).

Network of oscillators In some experiments, we use small networks of oscillators. Networks of oscillators can be built by introducing a functional coupling between oscillators, i.e. extending $\mathbf{p}$ by an additional summand comprised of the signals of the other oscillators.

$\mathbf{p}=\cdots+\sum_{j} \lambda_{j i} \mathbf{P}_{j i} \mathbf{R}_{j i} \mathbf{q}_{j}$ 
here $\lambda_{i} j$ is the strength of coupling from oscillator $j$ to oscillator $i, \mathbf{P}$ is the, binary, coupling matrix and $\mathbf{R}$ is the rotation matrix (examples of the coupling will be given later).

$\mathbf{R}=\left(\begin{array}{cc}\cos \theta_{j i} & -\sin \theta_{j i} \\ \sin \theta_{j i} & \cos \theta_{j i}\end{array}\right)$

The use of harmonic oscillator makes it possible to design arbitrary phase relationships into networks of such oscillators. We use the method outlined in Buchli and Ijspeert (2004a) which is based on the idea that we can rotate the signal of an oscillator to introduce phase shifts. As shown in Buchli and Ijspeert (2004a) choosing $\theta$ is equivalent in specifying the phase shift between the oscillators.

So the complete expression reads

$$
\dot{\mathbf{q}}_{i}=\mathbf{F}_{H}\left(\mathbf{q}_{i}\right)+\underbrace{\sum_{j} \lambda_{j i} \mathbf{P}_{j i} \mathbf{R}_{j i} \mathbf{q}_{j}}_{\text {coupling }}+[\underbrace{K_{k, i} s_{k}(t)}_{\text {sensorinput }}, 0]^{T}
$$

\section{Hardware}

PUPPY II is a 8 DOF experimental quadruped robot designed by F. Iida (2005). For the purpose of this article, its main interesting characteristics are that it is under-actuated and has spring dynamics in the legs. See Fig. 1 for an illustration of the robot. We describe in turn the important characteristics of the robot.

Mechanical system Each leg has a rotational "hip" joint and a knee joint. Only the hip joint is actuated, by attaching the leg directly to a strong RC servo motor. The knee joint features a spring (cf. Fig. 1 for the geometry of the leg). Thus, the robot is underactuated, only 4 of the $8 \mathrm{DOF}$ are actuated and in addition, due to the springs, it has a very pronounced intrinsic dynamics in form of resonant frequencies. The weight of the robot is roughly $0.7 \mathrm{~kg}$. The robot is energetically not autonomous, the power is fed to the robot by a cable.

Sensors and motors The robot is outfitted with 4 different sensor modalities: Force sensitive resistors on the body of the robot and under the feet of the robot, 3-axis acceleration sensor, IR-position sensitive detector sensor, and potentiometers to measure the knee angles. In the presented work we use the knee angle sensors and the inertia sensor. Those sensors convey a lot of information about the movement of the robot. The motors of the robot are strong off-the-shelf $\mathrm{RC}$ servo motors which are controlled in position. a)

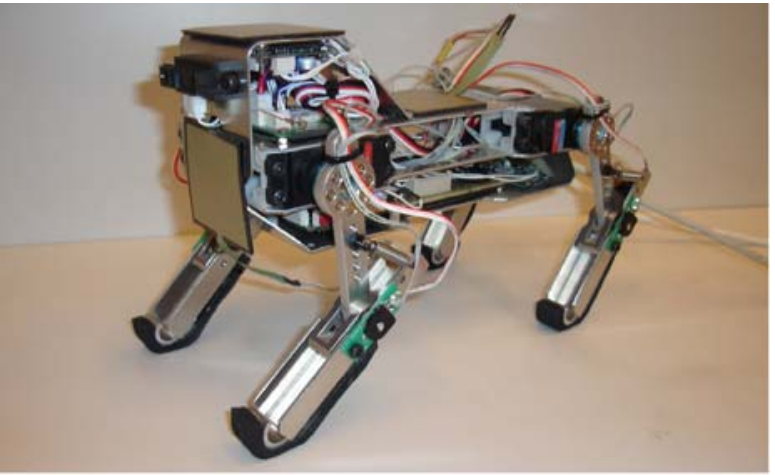

b)

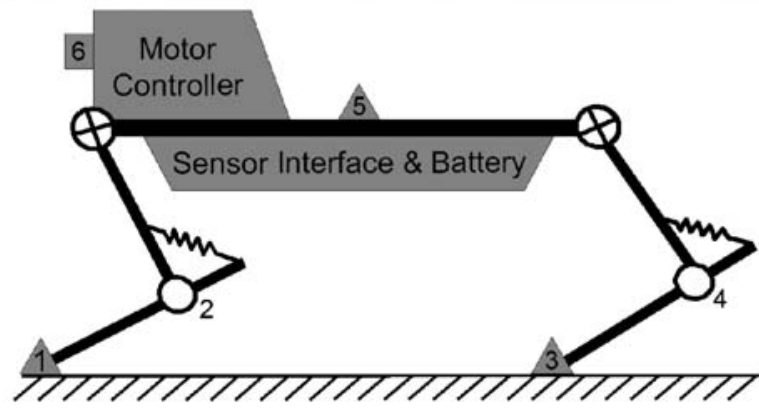

c)

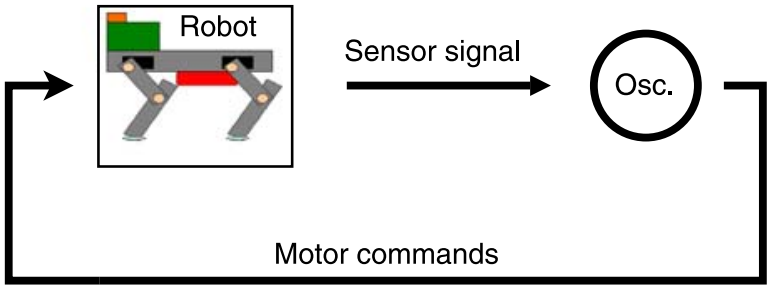

Fig. 1 (a) PUPPY II, a robot "dog" with passive dynamics (cf. springs in the knee joints) and several sensor modalities. (b) Mechanical structure of PUPPY II and sensor placement: 1, 3: FSR (Force Sensitive Resistors) 2, 4: Potentiometers of the passive joints 5: 3-axis acceleration sensors 6: PSD (Position Sensitive Detector). Circles with a cross denote actuated joints, blank circles denote passive joints. (c) Control structure used in the experiments: One of the sensor channels is used to perturb a controller consisting of one or several adaptive frequency Hopf oscillators, the output of the oscillator (a state variable) is used to send motor commands (position control). Thus, this system constitutes a nonlinear feedback loop

Control loop The oscillators are integrated with Euler integration on a off-board computer with a time-step of $t_{s}=10^{-3} \mathrm{~s}$. The sensor values are read and calculated setpoints are sent to the robot via the USB bus, with a sampling frequency of $50 \mathrm{~Hz}$, i.e. every 20th integration step the values are read out and the sensor values are updated. The faster integration step for the dynamical system is to ensure the numerical accuracy of the integration scheme.

The signal of the oscillator is converted into a desired angle for the leg by a linear transform

$\alpha_{t}=\alpha_{1} x(t)+\alpha_{0}$

where $\alpha_{t}$ is the desired angle for the leg, $\alpha_{0}$ is the center angle, $\alpha_{1}$ is the amplitude for a signal of amplitude 1. Later 
when presenting the results, we will list $\alpha_{1}$ and $\alpha_{0}$ for each experiment.

There is a low level controller (PD controller in the servos) which controls the position of the motor. The control performance of the low-level position control at the frequencies used for our experiments is sufficiently high that we can assume that the actual angle of the leg corresponds to the desired angle $\alpha_{t}$. The delay in the feedback loop is also sufficiently small that it does not pose an issue for the presented application.

Posture For each experiment we will characterize the setup of the robot in the following way (1) controller structure, i.e. number of oscillators, connection to sensors, motors and mutually (2) posture (cf. Fig. 2) of the robot and amplitude of movements for a signal of amplitude $x(t)=1$ and the center angle, i.e. the angle the leg assumes for a signal of amplitude $x(t)=0$. It is important to note these angles determine implicitly the posture of the robot, i.e. if the body is horizontal or more tilted forward or backwards.

Finally, in Fig. 3, we present an illustration of a typical gait of PUPPY II with the described controller and mechanical features. PUPPY II locomotes most efficiently (in terms

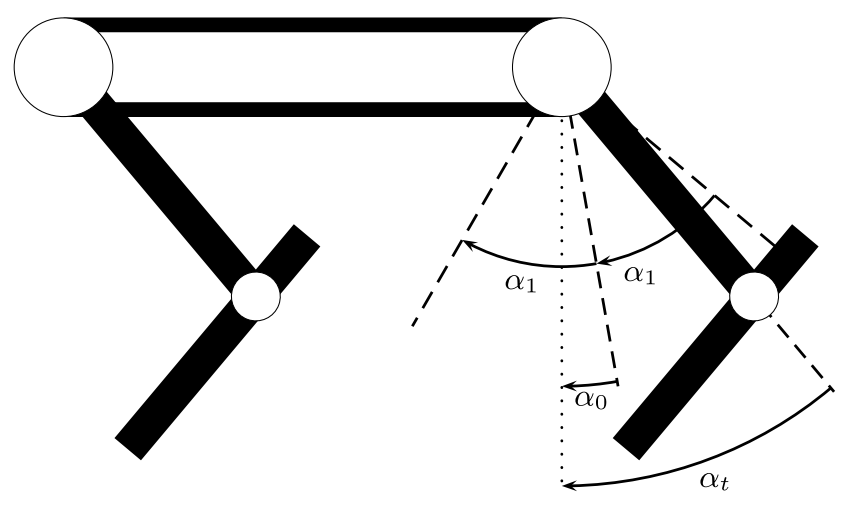

Fig. 2 Parameters describing the posture of the robot: center angle $\alpha_{0}$, i.e. angle for zero signal $x(t)=0$, angle $\alpha_{1}$ corresponding to a signal of amplitude $x(t)=1$. $\alpha_{t}$ is the actual position of the leg, $\alpha_{0}$ is the offset (compared to a vertical position) of the center position of the leg. Note that the vertical position denotes $\alpha_{0}=0$, legs titled backwards from this position have negative angles, and vice versa. This means the larger this angle, to more tilted forward is the leg. $\alpha_{1}$ is the amplitude of the leg for a signal of amplitude 1 (measured against $\alpha_{0}$ ) of forward velocity) with anti-phase (bounding) gaits but can also locomote with an in-phase (jump) gait.

\section{Experimental results}

In the following we describe several experiments which demonstrate some of the interesting features of the adaptive controller working together with the compliant robot. We use the experimental results to illustrate the two main results: (1) The adaptive controller is able to track the resonant frequency of the robot which is a function of different body parameters (2) controllers based on dynamical systems as we present are able to "recognize" mechanically intrinsic modes of locomotion, adapt to them and enforce them. These modes are expressed as phase relationships between the different limbs.

Further, we show the experimental evidence that the key properties of the gaits are not only depending on properties of the body but also the actual mode of movement that the body is operating in. And we show that even if we specify the gait pattern on the level of the CPG (by full coupling) the chosen gait pattern (measured by the foot-fall pattern) does not necessarily correspond to the CPG's pattern.

Experiment 1-Change of body properties In this experiment we demonstrate the capability of the dynamical system to constantly track the resonant properties, i.e. as soon as the body properties, here the weight, is changed the oscillators readjust the frequency to the new conditions.

The posture is: front legs $\alpha_{0}=14.38^{\circ}$, hind legs: $\alpha_{0}=$ $3.23^{\circ}$, the amplitudes for both pairs is $\alpha_{1}=10.8$. The controller setup is illustrated in Fig. 4 (left). It consists of a single oscillator which is connected to the output of the $z$-axis (vertical axis) of the inertia sensor.

To demonstrate the continuous adaptation capability, after the adaptation has converged, the weight of the robot is changed, what changes its resonant frequency. In Fig. 4 the data of the adaptation experiment is shown. It can be seen how immediately after the change of the body property the controller starts to tune to the new frequency. Later in the article we also present results with changed leg stiffness (cf. Experiment 5 and Fig. 11).
Fig. 3 Snapshots of a typical anti-phase gait of PUPPY II
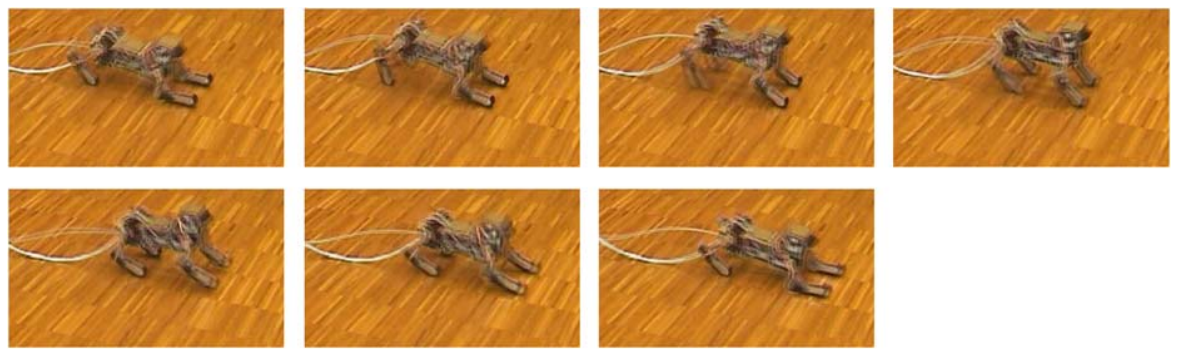


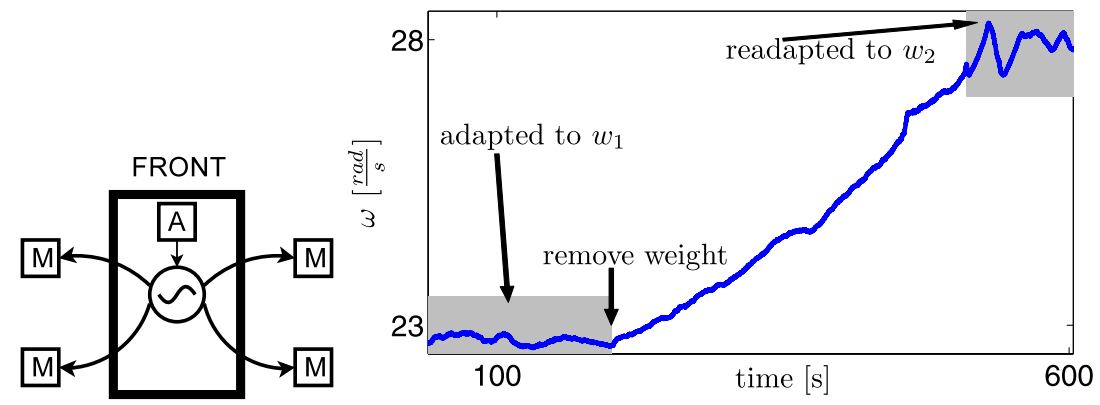

Fig. 4 Experiment 1 to show the online adaptation of the controller. On the left an illustration of the sensor-controller structure. The $z$-value of the acceleration sensor (A) is used as an input to the oscillator. The signal of the oscillator is used to set the motor position according to
(5). On the right the intrinsic frequency $\omega$ of the oscillator is shown as it evolves during the experiment. The body weight is changed from $m_{1}=0.905 \mathrm{~kg}$ to $m_{2}=0.695 \mathrm{~kg}$. The controller immediately starts to adapt to the changed body property
Fig. 5 (a) The range of posture angles used in the experiment illustrated on the robot. (b) The average frequency (squares) found by the adaptive frequency oscillator vs. posture, i.e. the angle of the leg. The bars show the standard deviation a)

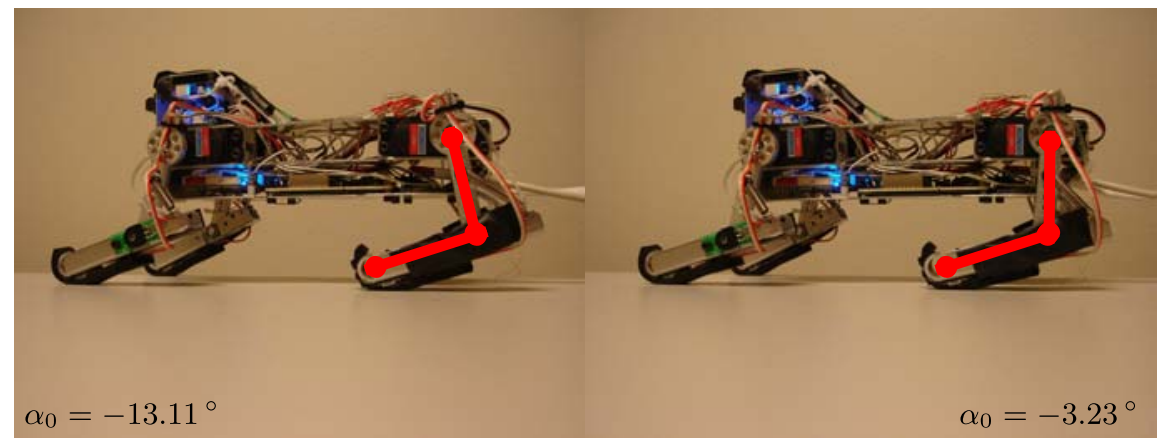

b)

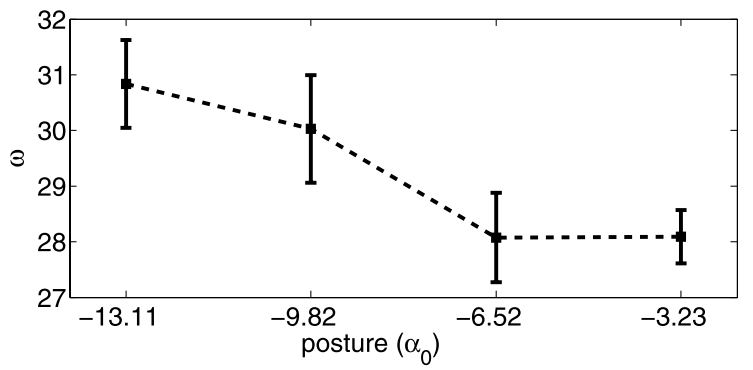

Experiment 2-Posture dependent frequency In this experiment we show that the frequency is not only dependent of obvious parameters like the weight of the robot but other, e.g. geometrical parameters, can influence the frequency as well. Here we show that it is dependent on the posture of the legs and the adaptive frequency oscillator is able to account for these different frequencies.

For this experiment the robot is again driven by a single oscillator and the inertia sensor is used as input to the oscillator (cf. Fig. 4a). While the front leg parameters are kept at $\alpha_{0}=14.38^{\circ}$ and $\alpha_{1}=10.80^{\circ}$, in this experiment the angle of the hind leg is varied from $\alpha_{0}=-13.11^{\circ}$ to $\alpha_{0}=-3.23^{\circ}$ while all the other parameters remain the same.
In Fig. 5 we show the average frequency which is assumed by the oscillator vs. the angle of the leg. As can be seen, the steeper the angle of the leg the higher the frequency found by the adaptation process.

By varying the posture angle $\alpha_{0}$ of the leg we vary the (average) incident angle of the foot which induces a change in the resonant frequency of the robot. As illustrated in Fig. 6 this makes sense considering the geometry of the leg: The steeper the leg the less of the force acting on the foot loads the springs and the larger becomes the force vector pushing along the axis of the foot and thus loading the motor. The force vector acting perpendicular (i.e. in direction of the free movement) to the lower leg gets smaller, thus there 
is less force loading the spring. The leg feels stiffer. Thus, the further the leg is tilted back, the stiffer the system be-

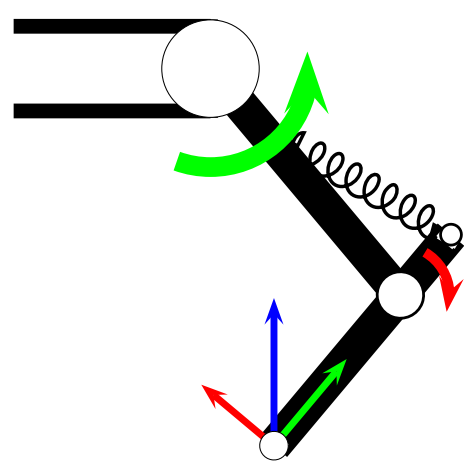

Smaller $\alpha_{0}$, steeper foot incident angle, higher stiffness, higher frequency

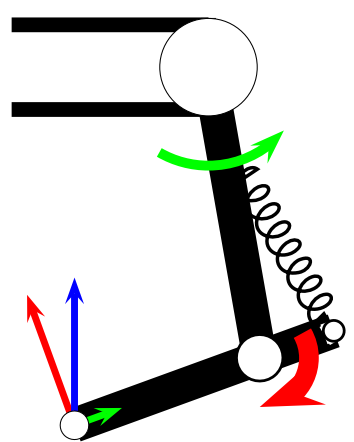

Larger $\alpha_{0}$, shallower foot incident angle, lower stiffness, lower frequency

Fig. 6 (Color online) Illustration of the effect of the posture angle $\alpha_{0}$ on the load distribution on the spring and motor respectively. Of the force acting on the leg (blue), only the part of the force active perpendicular to the foot loads the spring (red), the force acting parallel to the foot will act on the motor (green). Note that this is illustrative only for the principles at work, and that the real case is more complicated (e.g. amongst other things the force vector on the foot is not necessarily vertical)

a)

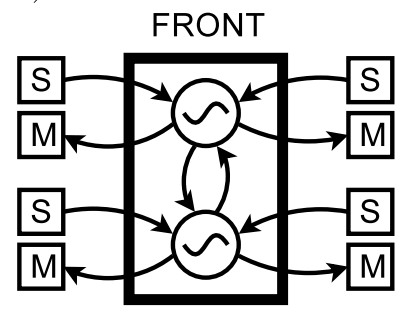

Fig. 7 Controller setup with two oscillators. (a) Setup with $\theta_{d}=0$, i.e. the two oscillator will synchronize in phase, this can also be interpreted as excitatory coupling as denoted by the arrow. (b) Setup with comes. Therefore, the frequency increases with decreasing $\alpha_{0}$ and vice versa.

Experiment 3-Gait dependent frequency In this experiment the robot is set up to assume two different gait patterns, by appropriate posture and the different controller setups.

In this experiment the legs are driven by two oscillators (illustrated in Fig. 7). Two distinct runs are made, one where the setpoints are given to the robot so that all the legs are in phase (Controller a, $\theta_{d}=0$ ) and the second one so that the hind-legs are in anti-phase with the front legs (Controller b, $\left.\theta_{d}=\pi\right)$.

Otherwise all the parameters of the robot are the same, especially the angles of the legs (i.e. the posture) to avoid effects on the tuning of the frequency of such parameters (as seen in the previous experiment).

In order to classify the gait pattern that the robot actually assumes we need to know when the feet touch the ground. In PUPPY II, we can infer the different phases (stance/swing) from the knee angle sensor. Since the springs are very stiff and the lower legs very light we can neglect dynamic effects of the rotation of the legs on the knee angle signal and assume that if the knee angle is not zero the leg is loaded, thus the leg is touching the ground.

As a control that the robot really assumes the gait pattern we want, in Fig. 8a we show the gait pattern for the two runs. We present the data as it is often presented in the biological/physiological literature, i.e. stance and swing phase. It gets clear that for the in-phase pattern the legs are active at the same time, while for the case when we invert the signal for the back legs we see a more alternating gait. In Fig. 8b the results of the two adaptation runs are presented, as can be seen the frequencies found are very distinct.

In order to investigate the reason for that its worthwhile looking at the gait pattern that the robot assumes in the two cases. The reason for the different frequencies stems from the fact that for the in phase gait, the springs of all four legs are loaded at more or less the same time, while for the bounding gait the springs of the front and hind legs are loaded more in an alternating fashion. This can be explained by a very simple spring mass model of the overall

$\theta_{d}=\pi$, i.e. the two oscillator will synchronize in anti-phase. This can also be interpreted as inhibitory coupling as denoted by the circle 
a)
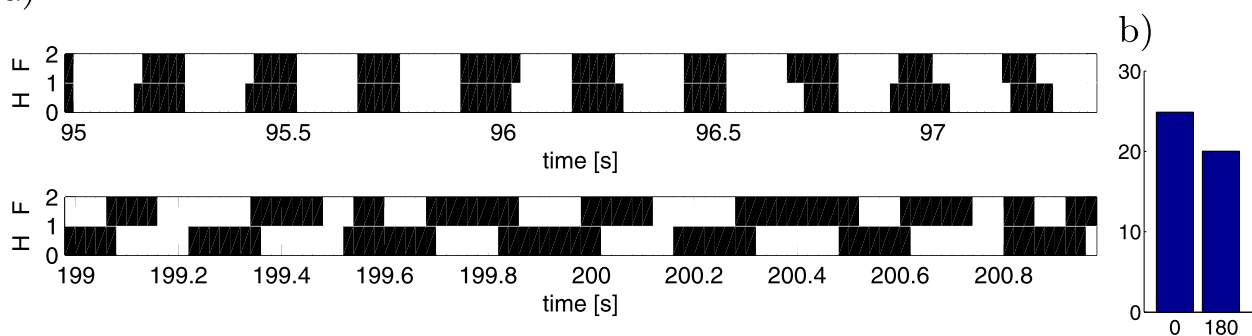

Fig. 8 Experiment to investigate the influence of the gait pattern on the resonant frequency. (a) Gait pattern, stance/swing phases, black is stance phase and white is swing phase, $\mathrm{H}$ depicts the hind legs and F depicts the fore legs, for simplicity only one leg per pair is shown, the data in each pair looks very similar. Top row for $\theta_{d}=0$, it is well

robot where we lump the springs of the legs into one single spring with a spring constant $k_{\text {eff }}$. If the springs of the legs are contracting at same time we have to add up the respective spring constants. Thus, for gaits where the legs are active at the same time the system behaves stiffer in average.

Note that the gait pattern does not exactly correspond to the CPG pattern (e.g. the gait pattern is not perfectly alternating in Fig. 8a bottom row). This will be studied in more details in the next experiment.

Experiment 4-Self-organization of gait: CPG pattern does not correspond to gait pattern The aim of this experiment is to show, that the gait pattern that the robot actually is assuming does not necessarily correspond to the gait pattern commanded by a CPG.

Again we use the same controller setup as before as in the last experiment with a single oscillator, and the two different phase settings. In this experiment we do not focus on the adaptation capability of the controller, but hold the frequency fixed at $\omega=20 \mathrm{rads}^{-1}$. The Posture of the robot is tilted forward by choosing a steeper angle for the back leg than for the front leg: hind $\alpha_{0}=-10.44^{\circ}, \alpha_{0}=10.06^{\circ}$. The amplitude is $\alpha_{1}=10.8^{\circ}$.

In Fig. 9 we present the gait patterns as estimated from the knee angles (in the same way as in the last experiment). First (in Fig. 9a), we present again the gait pattern by showing the stance and swing phases. Then, we present the time series of the phase difference of the two signals estimated with the Hilbert transform. We can see that if we specify a phase difference of $\pi$ for the CPG, we indeed get the expected result, a bounding gait where the front and the hind legs touch the ground with a phase difference of around $\pi$. However, if the phase difference for the CPG is specified as zero degrees, the robot does not assume a jumping gait in which both legs touch down in the same time, as one would expect. But, the phase difference estimated by the Hilbert transform varies mainly between $0.4 \pi$ and $\pi$, and in the stance-swing-diagram we can clearly see how the front legs visible that the front and hind limbs are active at the same time, while for $\theta_{d}=\pi$, we clearly see a alternating gait. (b) Resulting frequency of the adaptation process, the mean is at $\omega=24.89 \mathrm{rad} / \mathrm{s}$ for $\theta_{d}=\pi$ (alternating gait) and at $\omega_{d}=20.02 \mathrm{rad} / \mathrm{s}$
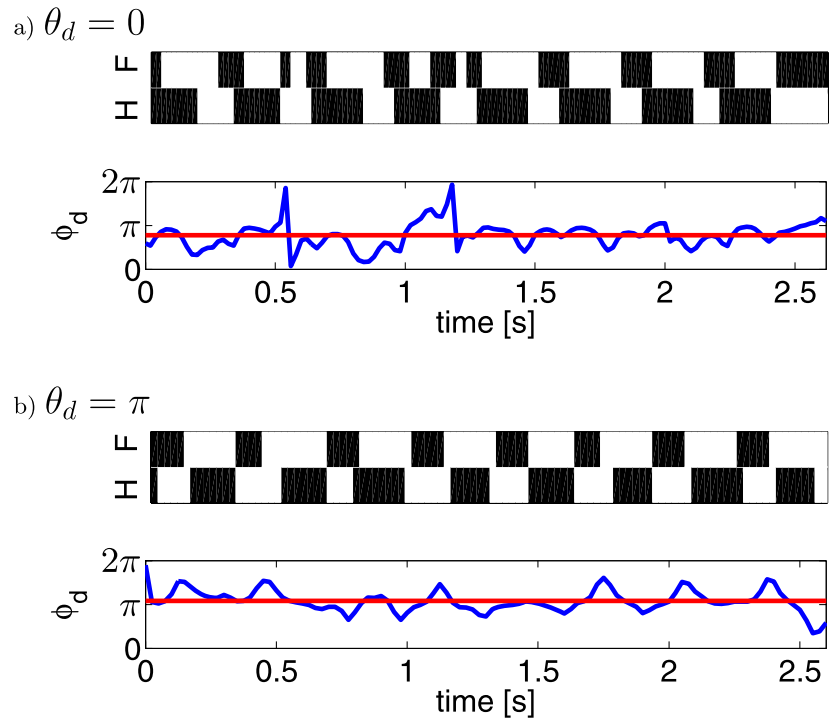

Fig. 9 (Color online) Gait pattern estimated by the knee angle data, black is stance and white is swing phase. (a) Coupling $\theta_{d}=0$, the first panel shows the gait pattern estimated by the knee angle data, the second panel shows the phase differences estimated by difference of the phase of the Hilbert transform of the knee angle data (red horizontal line indicates average difference). (b) The same information but this time the angle of the CPG is specified as $\theta_{d}=\pi$

touch down shortly after followed by a longer stance phase of the hind legs which initiates a flight phase. In other words, we command two very different patterns, but the body exhibits both times very similar gaits. Furthermore, we see that the gait pattern for $\theta_{d}=0$ is more irregular and more pseudo-periodic than the pattern for $\theta_{d}=\pi$, which means that this gait is less robust.

Also one has to note that the robot can be biased by tilting forward, i.e. for this experiment the robot posture is biased towards an anti-phase gait, while in the experiment before there was a more symmetrical posture which allow in-phase gaits to be mechanically stable. 
Fig. 10 Controller schema for Experiment 5. No connection between the oscillators for the hind and front leg

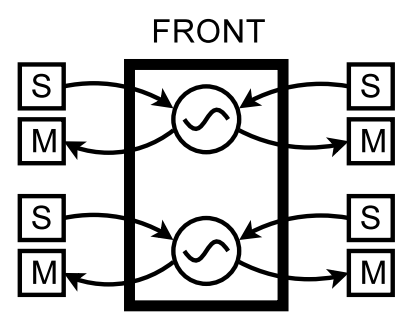

Experiment 5-Self-organization of gait pattern: Local adaptive controllers The aim of this experiment is to show, that we can exploit the tendency of the body of the robot to prefer certain gait patterns to use simpler, namely purely local control structures without direct inter-oscillator couplings. Motivated by the results of the previous experiment and earlier simulation studies (Buchli et al. 2005), namely the fact that the robot has modes which are mechanically more suitable, in this experiment we constrain the controllers of the robot less-there is no direct coupling between the oscillators for the hind and front legs. The only coupling that is established works through the mechanical system. As shown in Fig. 10 we use two oscillators, one for each pair of front and hind legs. The sensor feedback is the knee angle data from each leg. The oscillators are not directly coupled. The posture of the robot is as follows: hind leg $\alpha_{0}=-10.44^{\circ}$, front leg $\alpha_{0}=10.06^{\circ}$ and $\alpha_{1}=11.88^{\circ}$ for both pairs. Note that we have changed the stiffness of all legs, by attaching the spring differently, so that is has less lever. This makes the leg less stiff, and the frequency decreases. The reasons for this are twofold. First, to show yet another resonant frequency by changing the spring stiffness (as opposed to body mass as in Experiment 1). And secondly, the slower movements facilitate the experiment.

In Fig. 11a we show the frequency of the oscillators adapting to the body, and readapting after a perturbation. As can be seen in Fig. 11b, where we show the difference between the frequencies of the oscillators, despite the fact that they are not directly coupled, they remain very close. Furthermore, we show the phase difference in Fig. 11c, where it can be seen that the oscillators remain phase locked over the whole experiment. So the mechanical influence is enough to keep the oscillators phase locked and adapting in a coordinated fashion. Furthermore, it can be seen that the oscillators lock into an anti-phase pattern, what corresponds to the tendency for anti-phase gaits as found in the experiment before. We see that despite the freedom of the system and the loss of the specification of the phase difference, the system settles into a well coordinated movement, which corresponds to the mechanical "mode" of the robot. It can thus be said that this controller adapts to the mechanical properties of the body and current posture. We also see that the phase relationship is not exactly $\pi\left(\overline{\Delta \phi_{d}} \approx 1.05 \pi, \sigma\left(\Delta \phi_{d}\right) \approx 0.05 \pi\right)$, thus we see that a phase relationship which does not exactly correspond to $\pi$ seems more suited for the mechanical system in a)
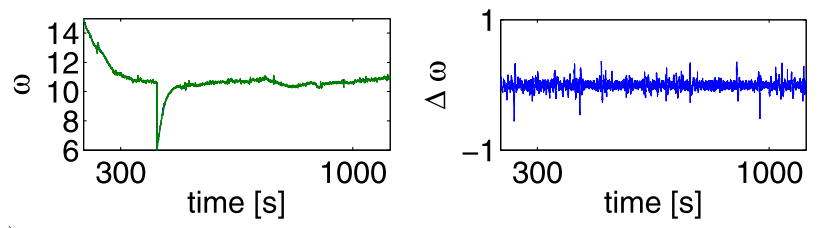

c)

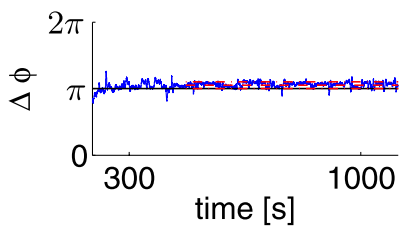

Fig. 11 Data from the adaptation experiment with local controllers (Experiment 5). (a) Adaptation of the frequencies, at around $400 \mathrm{~s}$ the frequencies are reset to $6 \mathrm{rads}^{-1}$. It is clearly visible that there exists an attractor for the convergence process are around $11 \mathrm{rads}^{-1}$. (b) The difference between the frequencies for the front and hind leg. It is clearly visible how closely together the two frequencies evolve. (c) The phase difference of the two oscillators, as can be seen they phase lock at a value slightly higher than $\pi\left(\overline{\Delta \phi_{d}} \approx(1.05 \pm 0.05) \pi\right.$, mean indicated by dashed lines, standard deviation dash-dot). And the phase locking is never lost during the whole experiments, i.e. the legs are always well coordinated

this setup since it is chosen by letting the body organizing the phase difference itself.

Thus, from this experiment we can conclude, that for coordinated motion, purely local controllers can be enough, i.e. mechanical coupling is enough to coordinate the different pairs of legs.

We can then ask, what is the need of direct coupling if local coupling seems to achieve the task. When trying out the local coupling with other postures than the one used in this experiment, it turns out the local coupling does not necessarily work for all postures (data not shown). However, we know from other experiments that they can provide locomotion if the phase difference is explicitely specified by inter-oscillator coupling. Thus, it turns out that we can make the gait more robust against this variation of posture, by employing a direct coupling.

To illustrate this with an example, consider the same experiment (local control) with another posture, a posture that tilts the body forward (e.g. as in Experiment 4). The two oscillators will converge to two different frequencies (data not shown). On the other hand the tilted position is good for efficient forward locomotion. We can thus imagine, that the controller learns about the appropriate gait pattern (i.e. the phase relationship found by local coupling) in a nontilted posture. After that the pattern is "fixed" by introducing a coupling which corresponds to the found mechanical gait pattern (i.e. by setting $\theta_{d}$ to the found value). Then, the body of the robot can be tilted forward to make it locomote with a gait pattern that efficiently makes use of the resonant dynamics (i.e. faster forward locomotion than if the robot would not use this gait pattern). 


\section{Toward a theory for AFOs in feedback loops}

So far we have presented empirical results, but it would be nice to be able to analyze what frequency the controller converges to and get a thorough understanding of the mechanism at work. For this we have to develop an understanding of AFOs in feedback loops. While in Righetti et al. (2006) the convergence behavior of the open loop case is analyzed we need to extend those results for the treatment of the closed loop case. As shown in Buchli et al. (2006a) for certain sensor modalities there is no convergence, but the frequency diverges. We need to develop an understanding for those cases. Thus, here we will show that we can understand the convergence behavior with the help of linear systems theory. In the following we will show the convergence analysis given a few assumptions hold.

For the following treatment it is convenient to write the Hopf oscillator, i.e. system (1), in the polar form, which is done by a straight forward transformation by substituting $\phi=\omega t$ and $r=\sqrt{q_{1}+q_{2}}$. We furthermore assume (without loss of generality) that the perturbation is $p=\left[p_{1}, 0\right]$. The system in the polar form reads

$\dot{r}=\left(\mu-r^{2}\right) r+\cos \phi p_{1}$

$\dot{\phi}=\omega-\frac{1}{r} \sin \phi p_{1}$

$\dot{\omega}=-\frac{1}{\tau} \sin \phi p_{1}$

\subsection{Adaptive Hopf oscillator with linear feedback loop}

In order to begin with the analysis of the system, the plant (i.e. the robot) will be modeled by a linear time-invariant system. This is a simplification (i.e. neglecting nonlinearities from the springs, kinematics of the body etc.), but, as we will see in the remainder of this article, this simplification yields already significant understanding of the mechanism of the adaptation process.

Thus, in the following we treat the "body" as a linear system. Therefore, let us assume the following systems: First, a linear $n$-th order SISO $^{1}$ system (the "robot", i.e. the motor commands are all lumped into one variable and only one sensor channel is modeled) of the form

$\dot{\boldsymbol{x}}=\mathbf{A} \mathbf{x}+\mathbf{B} u$

$y=\mathbf{C x}+\mathbf{D} u$

$\mathbf{A}$ is a $n \times n, \mathbf{B}$ a $n \times 1, \mathbf{C}$ a $1 \times n$ matrix, and $\mathbf{D}$ a scalar. $u$ is the (scalar) input to the linear system and $y$ the (scalar) output. Here, we can interpret $y$ as the sensory feedback and $u$ as the motor command.

\footnotetext{
${ }^{1}$ SISO: single-input-single-output.
}

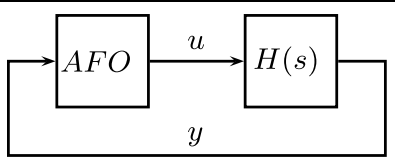

Fig. 12 Illustration of the feedback loop of the adaptive frequency oscillator with the linear system and the conventions used. The adaptive frequency oscillator (AFO) is connected to the plant, which is modeled as a linear system $H(s)$ through $u$ (the motor commands) and the sensory feedback from the robot to the AFO is modeled by $y$

Second, the adaptive frequency Hopf oscillator as described by (6)-(8). We set $u=r \cos \phi$ and $p_{1}=y$. Hence, the Hopf oscillator and the linear systems are connected in a feedback structure through their inputs $y$ (from the linear system to the Hopf oscillator) and $u$ (from Hopf to the linear system). See Fig. 12 for an illustration of the feedback structure.

As can be shown by linear systems theory, the linear system can not generate other frequencies than already present in the input $u$, it can however modify phase and amplitude of the signal. Therefore, we can write:

$y=M r \cos (\phi+\alpha)$

where $M=|H(s)|$ and $\alpha=\arg (H(s))(H(s)$ is the transfer function of the linear system).

We begin the analysis of the oscillator by writing the phase of the Hopf oscillator perturbed by a signal $y$

$\dot{\phi}=\omega-\frac{1}{r} \sin \phi y$

As outlined above we write $y(t)$ to be the state of the Hopf oscillator amplified by $M$ and rotated by $\alpha$

$y(t)=M r \cos (\phi+\alpha)$

thus

$\dot{\phi}=\omega-\sin \phi M \cos (\phi+\alpha)$

Using trigonometric transformations we can write this expression as

$\Longrightarrow \quad \dot{\phi}=\omega+\frac{1}{2} M[\sin \alpha-\sin (2 \phi+\alpha)]$

The results in Righetti et al. (2006) show that the systems shows a separation of timescale, i.e. the frequency adaptation process works on a timescale much slower than the convergence to the limit cycle with given frequency and radius. Thus, we assume $\omega=$ const and investigate what the observed frequency of the closed loops system will be. If this frequency is different from the intrinsic frequency, it should drive the slower adaptation process. $\omega=$ const also implies 
that $M$ and $\alpha$ are constants $(s=j \omega)$. We evaluate the average effective frequency $\bar{\Omega}$ with the given assumptions:

$\bar{\Omega}=\frac{1}{2 \pi} \int_{0}^{2 \pi} \omega+\frac{1}{2}[M \sin \alpha-\sin (2 \phi+\alpha)] d \phi$

Since by assumption $\omega$ is a constant and therefore $\alpha, M$ are constant, it follows that the first two summands are constants. The last summand is a $2 \pi$-periodic mean-free function, thus we get

$\bar{\Omega}=\omega+\frac{1}{2} M r \sin \alpha$

Let us define $\Delta \omega=\bar{\Omega}-\omega$, which is a useful notation to discuss convergence:

$\Delta \omega=\bar{\Omega}-\omega=\frac{1}{2} M r \sin \alpha$

As shown in Righetti et al. (2006), the slow variation of $\omega$ is as follows

$\Longrightarrow \quad \dot{\omega} \approx \frac{1}{2} M^{2} \frac{\omega}{\omega_{F}^{2}-\omega^{2}}$

where $\omega_{F}$ is the frequency of the rhythmic signal applied to the AFO. We can use this result to reason about the stability of the adaptation process. We use the fact that with the feedback loop $\omega_{F}=\bar{\Omega}$ and as we shown above this can be written as

$\omega_{F}=\omega+\Delta \omega$

Plugging this expression into (19) and assuming $\omega, \omega_{F}>0$ this means if $\Delta \omega>0, \omega$ increases, otherwise it decreases. If $\Delta \omega$ has a zero crossing with a negative slope $\left(\frac{\partial \Delta}{\partial \omega}<0\right)$, there is an attractive region around $\Delta \omega \approx 0$. Therefore, we expect the adaptation of the frequency to have a stable fixed point in this region. Hence, $\Delta \omega$ gives us information on the convergence of the system.

The magnitude of the linear system $M=|H(s)|$ can not be the determinant for convergence since it is positive, thus we have to focus on the phase of the linear system $\alpha=\arg H(s)$. We see that the term $\sin \alpha$ determines the zeros and the sign of $\Delta \omega$, conclusively the phase of the linear system $\alpha=\arg H(s)$ is the determinant for convergence of the adaptation process. In the following we illustrate the result with a simple linear system for two different "sensory channels".

\subsection{Stable at resonance}

Let us assume the following linear system:

$$
\mathbf{A}=\left[\begin{array}{cc}
0 & 1 \\
-\frac{k}{m} & -d
\end{array}\right]
$$

$\mathbf{B}=\left[\begin{array}{l}2 \\ 0\end{array}\right]$

$\mathbf{C}=[1,0]$

$\mathbf{D}=0$

This is 2 nd order (e.g. spring mass) system, which possesses a clear resonant frequency at $\omega_{r}=\sqrt{\frac{k}{m}}$. The values of the constants are largely irrelevant for the general result. For the presented data we have chosen $k=27^{2}, m=1$ and $d=0.1$. (Note that we use unit-less constants as their physical interpretation can vary.)

In Fig. 13, we present the Bode diagrams for the linear systems, and the result of the adaptation of oscillator. As discussed above, the bode plot already gives us a hint for stability of the adaptation process. More precisely we need to look at function $\Delta \omega$, but since this is a second order system, we know that the phase shift is maximum $2 \pi$, thus the phase can only have a single zero crossing. Since sinus is an odd function we also know that the sign does not change, thus we can read the stability of the adaptation directly out of the bode plot. In this example we see a negative zero crossing at the resonant frequency, thus we expect the adaptation process to converge to the resonant frequency.

In cases where we have a higher order linear system, and also for getting an idea about the quantitative behavior of the convergence, i.e. convergence rates, it is indeed helpful to look at the function $\Delta \omega$ which we obtain in a straight forward manner from the data in the bode plot and inserting into (17).

Thus, in this example, according to the peak in the convergence rate in Fig. 13c the convergence rate should increase and come to a sudden stop. This is indeed the case as can be seen in Fig. 13d, where we show the integration of the full system (6)-(10).

\subsection{Unstable at resonance}

Now, let us assume the same system as above but we change the coupling from the linear system to the Hopf oscillator (i.e. "change the sensor modality"), by setting

$\mathbf{C}=[0,0.1]$

i.e. the second state variable of the linear system is now used as input to the Hopf oscillator. Changing only the coupling means, the system has the same resonant frequency as before. Nevertheless, as we will see the adaptation does not converge to this frequency. Again, in Fig. 14, we present the Bode diagram. As before by looking at the phase of the linear system we already gain insight into the expected convergence properties of the frequency adaptation. This time there is no zero crossing, and the rate is positive. Thus, we expect 

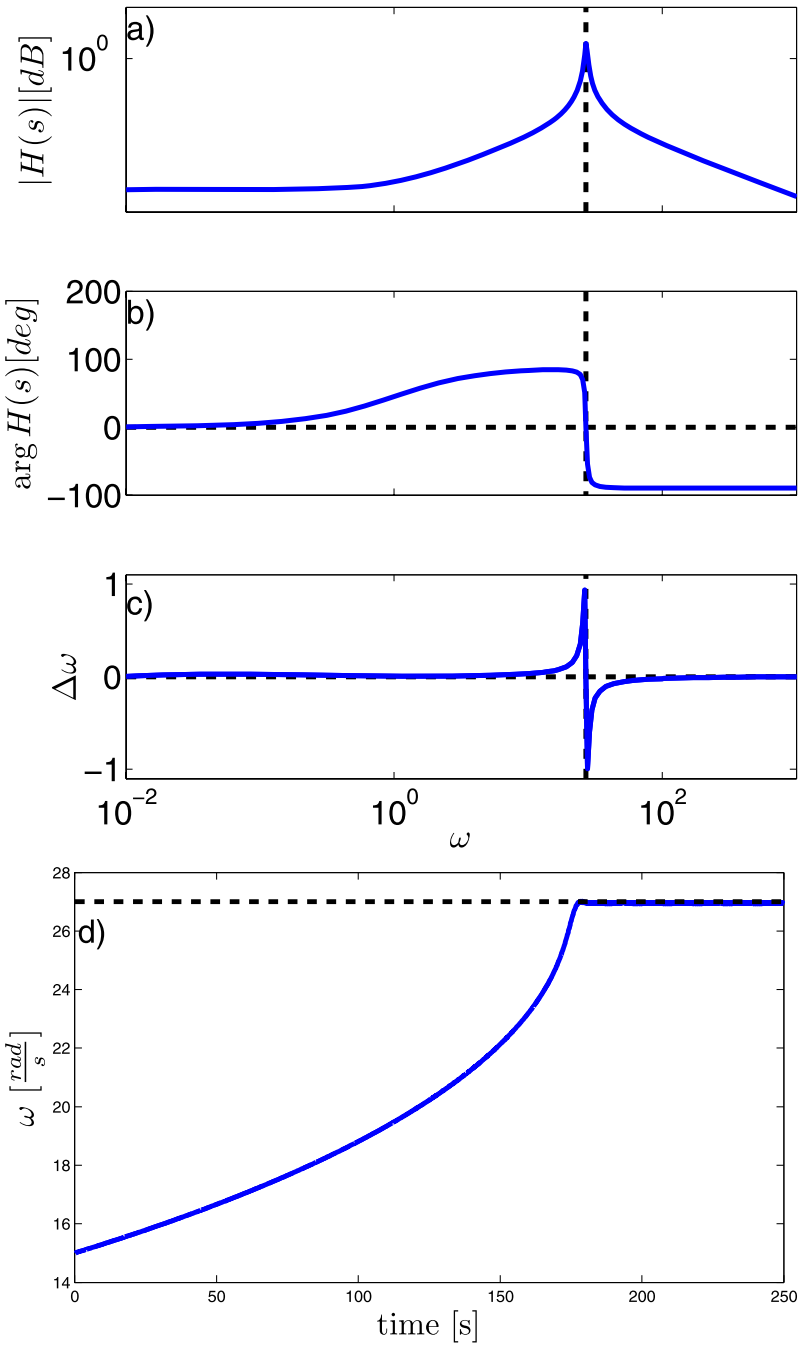

Fig. 13 A linear system for which the convergence is stable at resonance. (a), (b) Bode plot for $H(s)$, the dashed line indicates the resonant frequency. Note that the phase has a negative 0 crossing at the resonant frequency, this means the adaptation process has a attractor at the resonant frequency. (c) The function $\Delta \omega=\sin (H(s))$. Strictly speaking we need to look at this function to determine stability. (d) Time series of the integration of the full system showing the adaptation of the oscillator frequency $\omega$. It is clearly visible how the oscillator frequency adapts to the resonant frequency of the linear system (dashed line), what corresponds to the prediction from the Bode plot and $\Delta \omega$

the adaptation process to diverge. Looking at the function $\Delta \omega$, we can see that the convergence rate should be very low, but increases to a peak around the resonant frequency. And indeed, looking at Fig. 14c this prediction is confirmed. Instead of convergence, this means that $\omega$ crosses the resonant frequency with increased rate (Fig. 14d).

\subsection{Comparison with real world data}

In Fig. 15, we present data from the real robot for the two cases (convergence/divergence) and compare it with a fitted model of the basic system used before. The convergent
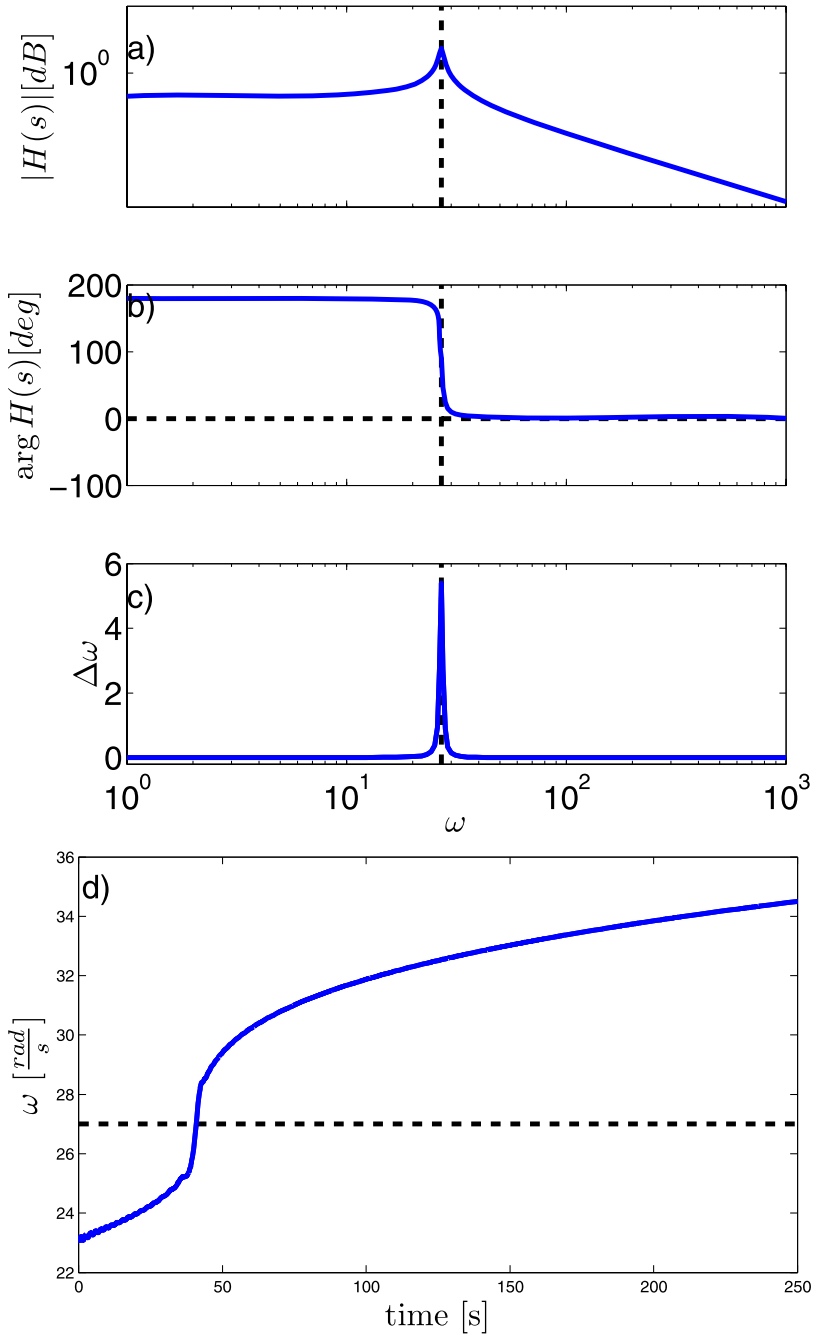

Fig. 14 A linear system for which there is no convergence at resonance. (a), (b) Bode plot for $H(s)$, note that the phase has no negative 0 crossing at the resonant frequency, this means the adaptation process has no attractor at the resonant frequency, and especially $\Delta \omega(\mathbf{c})$, is always positive with a distinct peak at the resonant frequency. This means divergence of the oscillator frequency $\omega$ with an increased rate to be expected around the resonant frequency. (d) Time series of $\omega$. The prediction is confirmed by the data obtained by the integration of the full system

behavior is observed by using the acceleration sensor, the divergent behavior can be observed by using the knee angle sensor instead. For the stable behavior (Fig. 15a) the data is $k=25.5^{2}, C=[0.45,0]$ and for the unstable behavior $k=28^{2}, C=[0,0.12]$ (Fig. 15b), all the other parameters are as above. As can be seen in the figures the linear systems reproduce qualitatively the data from the real robot. We are thus confident, that the linear treatment we presented conveys well the basic convergence properties of AFOs in feedback loops.

As we can see for the divergent case the match seems less good, however, already in the converging case we see the fact that the linear model predicts a higher convergence rate 


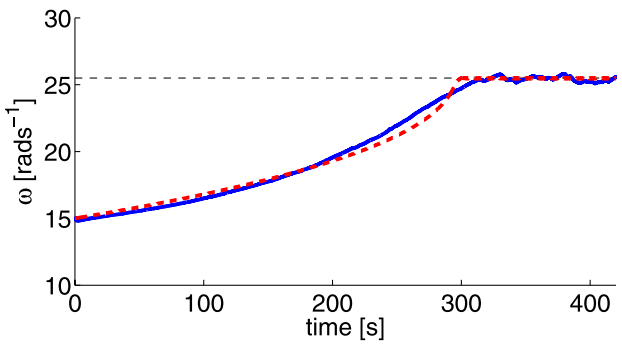

Fig. 15 Comparison of data from the real robot with the linear model. Left: sensor (acceleration) that leads to convergence. Right: sensor (knee angle) that does not lead to convergence. While the basic properties of the real world data are reproduced by the LTI model there

for regions close to the target frequency. This same effect leads to the mismatch between the linear model and reality in both cases only in the divergent case the effects become much more visible, since there is no fixed point behavior to which both solutions converge.

It is natural that the model can not fully predict the behavior close to the target frequency, since it was based on results which investigate the convergence behavior only for frequencies which are further away (cf. Righetti et al. 2006).

\subsection{From theory to design}

The developed theoretical insight can be used in the design of the robot or an experiment. If we understand the phase shift of the different sensory channels (either based on a model or on data) we can chose the sensor which will work best. Furthermore, if no sensor has the appropriate phase shift, then we can use filters to induce additional phase shifts and thus make convergence possible nevertheless.

\section{Conclusions and discussion}

We have shown five experiments from which several lessons can be learned and that help us to elucidate certain points which we deem very important for agile legged locomotion. They are the proof of concept of adaptive controllers which can track resonant frequencies of a body. The experiments give support to earlier proposed theoretical and simulation based work and the therein made assumptions by showing the application on a real robot. Also they highlight a few interesting facts about resonant frequencies in legged underactuated robots and the application of the CPG concept to such robots as we will describe in turn.

First, we have shown, that controllers based on adaptive frequency oscillators are able to track the resonant frequency of the robot. Different parameters can influence the frequency, such as mechanical parameters (weight, lengths,

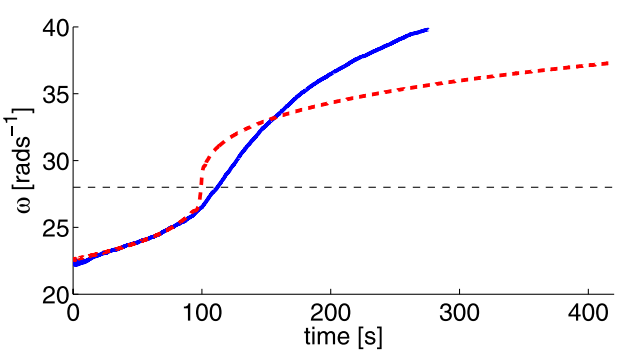

is some differences between the real data and the prediction of the LTI model. The dashed line indicates the estimated resonant frequency. See text for further discussion

spring stiffness etc., cf. Experiments 1 and 5), but also geometrical "gait" parameters such as the angles of the legs as shown in Experiment 2.

Further, in Experiment 3 we have shown that the resonant frequency is different for different gaits: in-phase (jump) gaits have a lower frequency than out-of-phase gaits. While the explanation for this is rather simple, i.e. the co-activation of different numbers of springs for different gaits, this simple fact is not usually addressed in the discussion of resonant dynamics of legged locomotion and adaptive controllers.

Given all these many aspects (there are probably many more) that influence the resonant frequency, and given the fact that working in resonance is very important for efficient locomotion, it becomes clear that an adaptive controller is crucial. As we showed AFOs are a very suitable choice for finding resonance and we backed this findings up with the theoretical treatment.

In Experiment 4 we have shown that in a compliant robot, such as the presented one, the gait pattern (as measured by phase differences) exhibited by the mechanical system is not necessarily the same as the CPG pattern. This is a fact which is often overseen in the discussion of CPGs applied to robots (and animals). This is in contrast to design methodologies which are aimed at high-gain fully actuated non-compliant robots, where it is made sure that the commanded trajectories are accurately followed and influences from body dynamics are not desired. In the case of an underactuated compliant robot as presented here, the physics of the body has to be accounted for, including the fact that we have distinct flight and stance phases in which the underactuated system reacts differently etc. We can exploit this organizational capability of the robot for certain situations, i.e. to achieve efficient self-organized locomotion with a minimum of control effort.

Consequently, in Experiment 5 we show that controllers built with well suited dynamical system which are adaptive and not over-specified can adapt to the body's "preferred" locomotion modes. With this experiment we also showed, that the inter-controller communication is not always mandatory, but mechanical coupling can be enough 
(see also Cruse et al. 1994; Kuniyoshi and Suzuki 2004). This fact could be interesting in the framework of modular or ad-hoc robots.

In Buchli and Santini (2005) it has been proposed to give the systems freedom to self-organize on certain variables of the system (i.e. the phase in our case), i.e. pull out control (in the appropriate way). Here we do exactly that: normally the oscillators are coupled, we removed this coupling and show that the system nevertheless finds good locomotion, in this sense the presented system is an application example of the proposed concepts and the results a proof of concept.

While there are many reports that biological motor systems are self-organizing (Kelso and Jeka 1992; Haken et al. 1985; Kelso et al. 1979; Schöner et al. 1990; Schöner and Kelso 1988) we have no methodology at hand for synthesizing such a system or its controller. Ideally we would like the system to be able to recognize, learn and adapt to different possibly co-existing modes. For this, traditional control theory is not of big help as it deals mostly with linear (or linearized) systems, in which no self-organization can take place. To develop such adaptive controllers which can find and switch between modes, the normal control engineering approach does not work because it usually involves a linearizing step after modeling. By this step we remove all the other attractors in the system, thus a controller which is developed based on this model can evidently not exploit different modes.

Arriving with such controllers would mean that we could build systems which are less frustrated (i.e. where the activation works in step with the body dynamics instead of forcing "artifical" patterns on the body). Non-frustrated systems mean also less energy consumption, higher efficiency (cf. Buchli et al. 2005) less mechanical stress, wear and tear etc. In Experiment 3 (Fig. 8) and Experiment 4 (Fig. 9) we note that if the system is specified against its "natural"/intrinsic modes, the gaits are less regular and less robust. Thus, it is important that the controller works with the body.

In a second part of the article we have laid out the theoretical treatment of AFOs in feedback loops and have compared the results of a simple linear model with data from the real robot. This comparison shows that even the simplest spring mass model can account quite well for the convergence effects that are observed. The theoretical treatment of AFO in feedback loops together with a more complete model of the robot (i.e. where the posture angles show up as parameters) will allow to understand most of the effects seen in the experiment in a straight forward manner.

The theoretical treatment shows that the convergence property is determined by a very fundamental property found in dynamical systems, namely the phase shift. Thus, AFOs are very generally applicable to work with resonant properties of systems: adaptive frequency oscillators in feedback loops can be used to either tune to or avoid to reso- nant frequency of a system irrespective of the implementation details of the system. Elsewhere we reported the use of feedback coupled AFOs for frequency analysis of signals (Buchli et al. 2008) and learning of structurally stable limit cycles (Righetti and Ijspeert 2006). As for the frequency adaptation mechanism itself, we exploit such a $b a$ sic and fundamental dynamic mechanism in oscillators that it would be a surprise that this is not a mechanism which is of more generic use (and is exploited in natural systems), yet the mechanism is rather novel and more examples of its use and applicability have to be found. The second mechanism we exploited namely phase synchronization is also a very general property of oscillators. The interesting result is that phase synchronization can be established via the mechanical coupling alone and that the established phase shift corresponds to a forward gait.

Future work We can conclude from such experiments that compliant robots offer both opportunities and challenges for control. There are two main areas where we lack support for usable, agile robotic legged locomotion. First in the area of actuators, and second in the control methodology. While compliance offers us nice body modalities and easy to achieve robust locomotion, such a robot as presented here definitely lacks certain controllability which severely restricts its practical use. It is a fundamental theoretical fact, that elastic modes decrease the controllability of the plant. In other words, as an example, we can not achieve exact foot placement with this robot. Actuators with controllable stiffness, as we see implemented in animals, would allow us to use the advantages of both high accurate control and compliant mechanics. In the beginning it would already be interesting just to experiment with actuators which can switch from passive to active mode. Stiffness control would allow precise control for some tasks while allowing self-organized movements for other tasks. Such actuators are not a niceto-have feature but an absolute must if we ever want to achieve the flexibility and agility with our robot as observed in their natural counterparts. The theoretical aspects of stiffness control, or more general impedance control is already being investigated for robotic tasks which involve contact with the environment (Hogan 1985; Valency and Zacksenhouse 2003) and recently also for legged robots with elastic actuators (Pratt et al. 2004). It will be interesting to bring together those results with adaptive controllers as presented here.

Then, as for the methodology to develop such adaptive controllers and the applied concepts, it sums up to the challenges for controller design for self-organizing systems, i.e. controllers which can make use of the self-organizing capabilities of the system to be controlled (Buchli and Santini 2005). Ideally we would like to have a controller which is able to learn about the system to be controlled. A controller which can learn about the intrinsic modes, i.e. that 
feels the intrinsic patterns formed by the underlying system, can modulate, enforce, stabilize or destabilize those modes depending on specified goals. In the here presented system, one of these modes is for example the coordination between front and hind legs, i.e. the phase relationship, another one the resonant frequency.

One has to realize that locomotion is always about cyclic movement and coordination (i.e. determining the phase difference) of different degrees of freedom. In fact the discussion can be cast in very formal language by methods based on Differential Geometry (cf. Marsden and Ostrowski 1998; Shammas et al. 2007a, 2007b). While the application of this framework to the here presented result is clearly out of the scope of this article, future work will have to address modeling of our system with these methods in order to achieve a fundamental understanding of the complete system.

In summary, to achieve agile legged robotic locomotion we need

- An appropriate body: suitable mechanics, compliant, with stiffness controlled actuators.

- An appropriate controller which can coordinate with the body and other controllers. The controller needs to be able to adapt to body and environment.

- Appropriate sensors. In order that the controller can get information about the body and its state the robot has to be equipped with appropriate sensors. Interestingly, it turns out that many sensor convey information about the intrinsic modes. It will however be interesting to explore to what extent redundant sensory information can be exploited to extract more meaningful information.

We showed one building block for such controllers with interesting results and a way how to approach the problem but a lot of work needs to be done before we reach our goal.

Thus in future, the presented research needs to be put forward on the identified two main axes, mechanics and control.

(1) Mechanics-Develop and test systems where we can switch from one control mode to the other, i.e. from high gain to compliant.

(2) Theory of control-We have laid out the theoretical fundament for the understanding of adaptive frequency oscillators in feedback loops. The next large step for the theoretical treatment is a generalization of the understanding to multiple-input-multiple-output (MIMO) systems. Furthermore, to advance the theoretical understanding in a next step a comprehensive model of the robot should be developed and the theory applied to this model. More generally we have to put forward a theory of control for self-organizing systems as discussed above.

As a more concrete example, it will be interesting to investigate how such controllers can be combined with the control of speed and direction, and how to resolve possibly conflicting goals within the controller architecture. Preliminary studies indicate that control of speed and direction can be achieved in a very straight forward manner by modulating the amplitudes or phase shifts on one side of the robot.

In addition to the presented ones we have observed many more interesting phenomena, such as higher periods in gaits and bistability amongst other dynamic effects, which will be the focus of future experiments. However, the description of phenomena will take more data gathering, amongst others with a motion tracking system.

Acknowledgements This work is funded by a Young Professorship Award to Auke Ijspeert from the Swiss National Science Foundation.

We would like to thank Fumiya Iida to make PUPPY II accessible for our research.

\section{References}

Arnol'd, V. I. (1983). Remarks on the perturbation theory for problems of mathieu type. Russian Mathematical Surveys, 38(4), 215-233.

Buchli, J., \& Ijspeert, A. J. (2004a). Distributed central pattern generator model for robotics application based on phase sensitivity analysis. In A. J. Ijspeert, M. Murata, \& N. Wakamiya (Eds.), Lecture notes in computer science: Vol. 3141. Biologically inspired approaches to advanced information technology: first international workshop, BioADIT 2004 (pp. 333-349). Berlin: Springer.

Buchli, J., \& Ijspeert, A. J. (2004b). A simple, adaptive locomotion toy-system. In S. Schaal, A. J. Ijspeert, A. Billard, S. Vijayakumar, J. Hallam, \& J. A. Meyer (Eds.), From animals to animats 8. Proceedings of the eighth international conference on the simulation of adaptive behavior (SAB'04) (pp. 153-162). Cambridge: MIT Press.

Buchli, J., \& Santini, C. C. (2005). Complexity engineering: Harnessing emergent phenomena as opportunities for engineering. In $R e$ ports of the Santa Fe Institute's complex systems summer school 2005, Santa Fe Institute.

Buchli, J., Righetti, L., \& Ijspeert, A. J. (2005). A dynamical systems approach to learning: a frequency-adaptive hopper robot. In Lecture notes in artificial intelligence. Proceedings of the VIIIth European conference on artificial life ECAL 2005 (pp. 210-220). Berlin: Springer.

Buchli, J., Iida, F., \& Ijspeert, A. J. (2006a). Finding resonance: Adaptive frequency oscillators for dynamic legged locomotion. In Proceedings of the IEEE/RSJ international conference on intelligent robots and systems (IROS) (pp. 3903-3909). New York: IEEE Press.

Buchli, J., Righetti, L., \& Ijspeert, A. J. (2006b). Adaptive frequency oscillators applied to dynamic walking II. Adapting to resonant body dynamics. In Proceedings of dynamic walking. Abstract.

Buchli, J., Righetti, L., \& Ijspeert, A. J. (2006c). Engineering entrainment and adaptation in limit cycle systems-from biological inspiration to applications in robotics. Biological Cybernetics, 95(6), 645-664.

Buchli, J., Righetti, L., \& Ijspeert, A. (2008, in press). Frequency analysis with coupled nonlinear oscillators. Phyisca D.

Cruse, H., Brunn, D. E., Bartling, C., Dean, J., Reifert, M., Kindermann, J., \& Schmitz, T. (1994). Walking: A complex behavior controlled by simple networks. Adaptive Behavior, 3(4).

Crespi, A., \& Ijspeert, A. J. (2006). AmphiBot II: An amphibious snake robot that crawls and swims using a central pattern generator. In Proceedings of the 9th international conference on climbing and walking robots (CLAWAR 2006) (pp. 19-27). 
Cham, J. G., Karpick, J. K., \& Cutkosky, M. R. (2004). Stride period adaptation of a biomimetic running hexapod. International Journal of Robotics Research, 23(2), 141-153.

Collins, J. J., \& Richmond, S. A. (1994). Hard-wired central pattern generators for quadrupedal locomotion. Biological Cybernetics, 71(5), 375-385.

Collins, S., Ruina, A., Tedrake, R., \& Wisse, M. (2005). Efficient bipedal robots based on passive-dynamic walkers. Science, 307(5712), 1082-1085.

Endo, G., Nakanishi, J., Morimoto, J., \& Cheng, G. (2005). Experimental studies of a neural oscillator for biped locomotion with QRIO. In Proceedings of the 2005 IEEE international conference on robotics and automation, Barcelona, Spain (pp. 598-604).

Fischer, M. S., \& Blickhan, R. (2006). The tri-segmented limbs of therian mammals: kinematics, dynamics, and self-stabilizationa review. Journal of Experimental Zoology, 305A(11), 935-952.

Futakata, Y., \& Iwasaki, T. (2008, in press). Formal analysis of resonance entrainment by central pattern generator. Journal of Mathematical Biology. Available online.

Full, R. J., \& Koditscheck, D. E. (1999). Templates and anchors: neuromechanical hypotheses of legged locomotion on land. Journal of Experimental Biology, 202, 3325-3332.

Grillner, S. (1985). Neurobiological bases of rhythmic motor acts in vertebrates. Science, 228(4696), 143-149.

Geyer, H., Seyfarth, A., \& Blickhan, R. (2004). Spring-mass running: simple approximate solution and application to gait stability. Journal of Theoretiacl Biology, 232(3), 315-328.

Haken, H., Kelso, J. A. S., \& Bunz, H. (1985). A theoretical model of phase transitions in human hand movements. Biological Cybernetics, 51, 347-356.

Hogan, N. (1985). Impedance control-an approach to manipulation. $\mathrm{i}$ - theory. ii-implementation. iii-applications. Journal of Dynamic Systems, Measurement, and Control, 107, 1-24.

Hopf, E. (1942). Abzweigung einer periodischen Lösung von einer stationären Lösung eines Differentialsystems. Ber. Math.-Phys., Sächs. Akad. d. Wissenschaften, Leipzig (pp. 1-22).

Iida, F. (2005). Cheap design approach to adaptive behavior: Walking and sensing through body dynamics. In International symposium on adaptive motion of animals and machines.

Iida, F., Gomez, G. J., \& Pfeifer, R. (2005). Exploiting body dynamics for controlling a running quadruped robot. In Proceedings of the 12th int. conf. on advanced robotics (ICAR05) (pp. 229-235).

Ijspeert, A. J. (2001). A connectionist central pattern generator for the aquatic and terrestrial gaits of a simulated salamander. Biological Cybernetics, 84(5), 331-348.

Ijspeert, A., Crespi, A., Ryczko, D., \& Cabelguen, J.-M. (2007). From swimming to walking with a salamander robot driven by a spinal cord model. Science, 315(5817), 1416-1420. Additional material, like the supporting online material, movies and pictures can be accessed via the. $\mathrm{html}$ link.

Iwasaki, T., \& Zheng, M. (2006). Sensory feedback mechanism underlying entrainment of central pattern generator to mechanical resonance. Biological Cybernetics, 94(4), 245-261.

Kimura, H., \& Fukuoka, Y. (2004). Biologically inspired adaptive dynamic walking in outdoor enviornment using a self-contained quadruped robot: Tekken2. In Proceedings IROS 2004.

Kimura, H., Fukuoka, Y., \& Cohen, A. V. (2004). Biologically inspired adaptive dynamic walking of a quadruped robot. In S. Schaal, A. J. Ijspeert, A. Billard, S. Vijayakumar, J. Hallam, \& J. A. Meyer (Eds.), From animals to animats 8. Proceedings of the eighth international conference on the simulation of adaptive behavior (SAB'04) (pp. 201-210). Cambridge: MIT Press.

Kelso, J. A. S., \& Jeka, J. J. (1992). Symmetry breaking dynamics of human multilimb coordination. Journal of Experimental Psychology, 18(3), 645-668.
Kelso, J. A. S., Southard, D. L., \& Goodman, D. (1979). On the nature of human interlimb coordination. Science, 203(4384), 10291031.

Kuniyoshi, Y., \& Suzuki, S. (2004). Dynamic emergence and adaptation of behavior through embodiment as coupled chaotic field. In Proceedings of 2004 IEEE/RSJ international conference on intelligent robots and systems (pp. 2042-2049). New York: IEEE Press.

McGeer, T. (1990). Passive dynamic walking. International Journal of Robotics Research, 9, 62-82.

Morimoto, J., Endo, G., Nakanishi, J., Hyon, S., Cheng, G., Bentivegna, D., \& Atkeson, C. G. (2006) Modulation of simple sinusoidal patterns by a c oupled oscillator model for biped walking. In Proceedings of the 2006 IEEE international conference on robotics and automation (pp. 1579-1584), May 2006.

Marsden, J. E., \& Ostrowski, J. (1998). Symmetries in motion: Geometric foundations of motion control. Nonlinear Science Today.

Pikovsky, A., Rosenblum, M., \& Kurths, J. (2001). Cambridge nonlinear science series: Vol. 12. Synchronization, a universal concept in nonlinear sciences. Cambridge: Cambridge University Press.

Pratt, G. A., Willisson, P., Bolton, C., \& Hofman, A. (2004). Late motor processing in low-impedance robots: Impedance control of series elastic actuators. In Proceedings of the 2004 American control conference (pp. 3245-3251).

Raibert, M. H. (1990). Trotting, pacing and bounding by a quadruped robot. Journal of Biomechanics, 23, 79-98.

Raibert, M., \& Hodgins, J. K. (1993). Legged robots. In Biologicial neural networks in invertebrate neuroethology and robotics (pp. 319-354). San Diego: Academic Press.

Reddy, D. V. R., Sen, A., \& Johnston, G. L. (2000). Dynamics of a limit cycle oscillator under time delayed linear and nonlinear feedbacks. Physica D, 144(3-4), 335-357.

Righetti, L., \& Ijspeert, A. J. (2006). Programmable central pattern generators: an application to biped locomotion control. In Proceedings of the 2006 IEEE international conference on robotics and automation.

Righetti, L., Buchli, J., \& Ijspeert, A. J. (2006). Dynamic hebbian learning in adaptive frequency oscillators. Physica D, 216(2), 269-281.

Shammas, E. A., Choset, H., \& Rizzi, A. A. (2007a). Geometric motion planning analysis for two classes of underactuated mechanical systems. The International Journal of Robotics Research, 26(10), 1043-1073.

Shammas, E. A., Choset, H., \& Rizzi, A. A. (2007b). Towards a unified approach to motion planning for dynamic underactuated mechanical systems with non-holonomic constraints. The International Journal of Robotics Research, 26(10), 1075-1124.

Schöner, G., \& Kelso, J. A. S. (1988). Dynamic pattern generation in behavioral and neural systems. Science, 239(4847), 1513-1520.

Schöner, G., Jiang, W. Y., \& Kelso, J. A. S. (1990). A synergetic theory of quadrupedal gaits and gait transitions. Journal of Theoretical Biology, 142, 359-391.

Tsuchiya, K., Aoi, S., \& Tsujita, K. (2002). Locomotion control of a multi-legged locomotion robot using oscillators. In 2002 IEEE intl. conf. SMC (Vol. 4).

Verdaasdonk, B. W., Koopman, H. F. J. M., \& van der Helm, F. C. T. (2007). Resonance tuning in a neuro-musculo-skeletal model of the forearm. Biological Cybernetics, 96(2), 165-180.

Valency, T., \& Zacksenhouse, M. (2003). Accuracy/robustness dilemma in impedance control. Journal of Dynamic Systems, Measurement, and Control, 125, 310-319. 


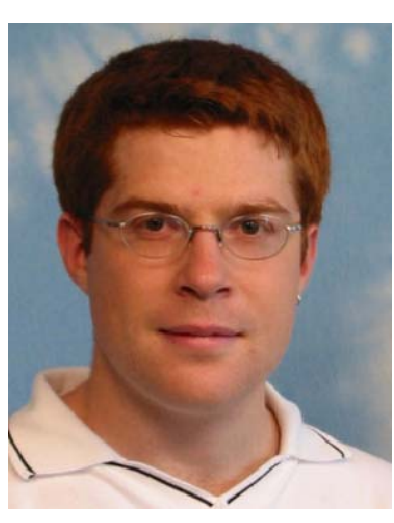

Jonas Buchli is currently a Postdoctoral Fellow at the Computational Learning and Motor Control Lab. at the University of Southern California in Los Angeles. He received a Diploma in Electrical Engineering from the ETHZ (the Swiss Federal Institute of Technology, Zürich) in 2003 and a Doctorate from the EPFL (the Swiss Federal Institute of Technology, Lausanne) in 2007. His research interests include self-organization and emergent phenomena in complex systems, the theory of nonlinear dynamical systems and information concepts. And, especially the possible applications of these topics to the intersecting field of engineering and biology. He has been involved in the organization of several international conferences and is reviewer for international conferences and journals.

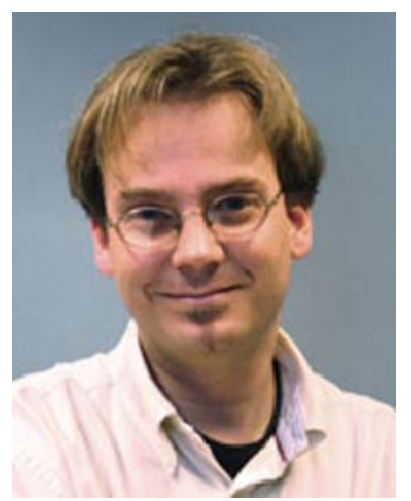

Auke Jan Ijspeert is an assistant professor at the EPFL (the Swiss Federal Institute of Technology at Lausanne), and head of the Biologically Inspired Robotics Group (BIRG). He has a B.Sc./M.Sc. in physics from the EPFL, and a Ph.D. in artificial intelligence from the University of Edinburgh. His research interests are at the intersection between robotics, computational neuroscience, nonlinear dynamical systems, and machine learning. $\mathrm{He}$ is interested in using numerical simulations and robots to get a better understanding of the sensorimotor coordination in animals, and in using inspiration from biology to design novel types of robots and adaptive controllers (see for instance Ijspeert et al., Science 315(5817):1416-1420, 2007). With his colleagues, he has received the Best Paper Award at ICRA2002, and the Industrial Robot Highly Commended Award at CLAWAR2005. He was the Technical Program Chair of 5 international conferences (BioADIT2004, SAB2004, AMAM2005, BioADIT2006, LATSIS2006), and has been a program committee member of over 25 conferences. For more info see: http://birg.epfl.ch. 